INPLASY

PROTOCOL

To cite: Li et al. Acupuncture combine with tuina for diabetic peripheral neuropathy:A protocol for a systematic review and meta-analysis. Inplasy protocol 2021110017. doi:

10.37766/inplasy2021.11.0017

Received: 05 November 2021

Published: 05 November 2021

Corresponding author:

Li Xuefeng

1414714202@qq.com

Author Affiliation:

Changchun University of

Chinese Medicine.

Support: (No.20190201147JC).

Review Stage at time of this submission: Preliminary

searches.

Conflicts of interest:

None declared.

\section{Acupuncture combine with tuina for diabetic peripheral neuropathy: A protocol for a systematic review and meta-analysis}

Li, X1; Wang, H2; Zhou, X³; Ma, D4; Chai, J5; Liu, J6; Qian, X77.

Review question / Objective: We aimed to perform a systematic review and meta-analysis to estimate the effectiveness of acupuncture combine with Tuina in DPN treatment.

Condition being studied: Diabetic peripheral neuropathy (DPN) is one of the most common microvascular complications of diabetes mellitus, with an incidence ranging from $60 \%$ to $90 \%$. With the change in modern dietary structure, the incidence of diabetes is increasing year by year, and DPN is also on the rise. Acupuncture and Tuina treatments are often combined to treat DPN; however, there has been no metaanalysis on their synergistic effect. Therefore, we aimed to perform a systematic review and meta-analysis to estimate the effectiveness of acupuncture combine with Tuina in DPN treatment.

INPLASY registration number: This protocol was registered with the International Platform of Registered Systematic Review and Meta-Analysis Protocols (INPLASY) on 05 November 2021 and was last updated on 05 November 2021 (registration number INPLASY2021110017).

\section{INTRODUCTION}

Review question / Objective: We aimed to perform a systematic review and metaanalysis to estimate the effectiveness of acupuncture combine with Tuina in DPN treatment.
Condition being studied: Diabetic peripheral neuropathy (DPN) is one of the most common microvascular complications of diabetes mellitus, with an incidence ranging from $60 \%$ to $90 \%$. With the change in modern dietary structure, the incidence of diabetes is increasing year by year, and DPN is also on the rise. 
Acupuncture and Tuina treatments are often combined to treat DPN; however, there has been no meta-analysis on their synergistic effect. Therefore, we aimed to perform a systematic review and metaanalysis to estimate the effectiveness of acupuncture combine with Tuina in DPN treatment.

\section{METHODS}

Participant or population: Diabetic peripheral neuropathy (DPN) is one of the most common microvascular complications of diabetes mellitus, with an incidence ranging from $60 \%$ to $90 \%$. With the change in modern dietary structure, the incidence of diabetes is increasing year by year, and DPN is also on the rise. Acupuncture and Tuina treatments are often combined to treat DPN; however, there has been no meta-analysis on their synergistic effect. Therefore, we aimed to perform a systematic review and metaanalysis to estimate the effectiveness of acupuncture combine with Tuina in DPN treatment.

Intervention: The treatment group using Tuina, while the control group received treatment with oral medication, acupuncture, Chinese herbal medication, physical therapy, Botox injections, and so on, or even with no treatment, will be included.

\section{Comparator: DPN patients.}

\section{Study designs to be included: RCTs.}

Eligibility criteria: We will consider patients with a clinical diagnosis of DPN irrespective of their gender, age, severity, and disease duration.

Information sources: Nine electronic databases were retrieved for this study. The English databases mainly retrieved PubMed, Web of Science, Embase, AMED, and the Cochrane Library, while the CNKI, VIP, CBM, and Wanfang databases were used to retrieve the Chinese literature. There is no definite time limit for the retrieval literature, and the languages are limited to Chinese and English. We will consider articles published between database initiation and October 2021. We used the Review Manager 5.4 software provided by the Cochrane Collaborative Network for statistical analysis. We then assessed the quality and risk of the included studies and observed the outcome measures.

Main outcome(s): The primary outcome included the glycemic profile, as measured by fasting blood glucose or glycated hemoglobin.

Additional outcome(s): The secondary outcomes consisted of neuropathic pain intensity, as assessed by visual analog scale or other relevant tools; plantar tactile sensitivity, as evaluated by SemmesWeinstein Monofilament Examination; sensory nerve conduction velocity and motor nerve conduction velocity, as assessed by electromyography; quality of life, as evaluated by health-related quality of life scale or associated scores, and adverse events.

Quality assessment / Risk of bias analysis: The literature quality of this study was evaluated using the bias risk table proposed by the Cochrane Collaborative Network. The risk table includes six items: random sequence generation mode, whether to use allocation concealment, whether to blind the subjects and intervention providers, whether to blind the results evaluators, whether the results data are complete, whether to select the results report, and other bias sources. The criteria used to assess the risk of bias were "low risk," "high risk," and "unclear." unclear. In this process, two evaluators independently evaluated methodological quality. In cases of disagreement, the third author intervened. If there are $>10$ trials in accordance with the study, we can use Rev Manager 5.4 software to draw and analyze the funnel chart, and use the funnel chart to evaluate the potential publication bias.

Strategy of data synthesis: We will use the Review Manager 5.4 software provided by the Cochrane Collaborative Network for 
statistical analysis. For continuous variables, the mean and SD of each study were obtained and pooled as mean difference (MD) or standardized mean differences (SMD) with a $95 \%$ confidence interval (CI). Statistical heterogeneity analysis was performed for the included clinical RCTs. The Cochrane 12 test was used for statistical analysis. When 12 was .05 , it indicated that there was no statistical heterogeneity between the studies, and the fixed-effect model was selected to combine the effect amount; otherwise, the random effect model was adopted.

Subgroup analysis: In the case of high heterogeneity, we conducted a subgroup analysis to identify the sources of heterogeneity. In addition, according to different course times, or other factors affecting the results, we will also perform a subgroup analysis.

Sensitivity analysis: To test the robustness of the main decisions in the review process, we conducted a sensitivity analysis. The main analysis points included the impact of method quality, sample size, and missing data on the study. The metaanalysis will be reused, and more inferiorquality studies will be excluded. The results were compared and discussed according to the results.

Country(ies) involved: China.

Keywords: acupuncture, tuina, diabetic peripheral neuropathy, effectiveness, safety, protocol, systematic review.

Contributions of each author:

Author 1 - Xuefeng Li.

Author 2 - Heran Wang.

Author 3 - Xue Zhou.

Author 4 - Dongyang Ma.

Author 5 - Jiapeng Chai.

Author 6 - Jiayi Liu.

Author 7 - Xin Qian. 